\title{
Robust Palmprint Verification Using 2D and 3D Features
}

\author{
David Zhang, Vivek Kanhangad, Nan Luo, Ajay Kumar \\ Biometrics Research Centre, Department of Computing, The Hong Kong Polytechnic \\ University, Kowloon, Hong Kong \\ \{csdzhang, csvivek, csnluo\}@comp.polyu.edu.hk, ajaykr@ieee.org
}

\begin{abstract}
This paper presents a new personal authentication system that simultaneously exploits 2D and 3D palmprint features. The objective of our work is to improve accuracy and robustness of existing palmprint authentication systems using 3D palmprint features. The proposed multilevel framework for personal authentication efficiently utilizes the robustness (against spoof attacks) of the 3D features and the high discriminating power of the 2D features. The developed system uses an active stereo technique, structured light, to simultaneously capture 3D image or range data and a registered intensity image of the palm. The surface curvature feature based method is investigated for 3D palmprint feature extraction while Gabor feature based competitive coding scheme is used for $2 \mathrm{D}$ representation. We comparatively analyze these representations for their individual performance and attempt to achieve performance improvement using the proposed multilevel matcher that utilizes fixed score level combination scheme to integrate information. Our experiments on a database of 108 subjects achieved significant improvement in performance with the integration of $3 \mathrm{D}$ features as compared to the case when $2 \mathrm{D}$ palmprint features alone are employed. We also present experimental results to demonstrate that the proposed biometric system is extremely difficult to circumvent, as compared to the currently proposed palmprint authentication approaches in the literature.
\end{abstract}

Keywords: Biometrics, palmprint verification, 3D palmprint features, Gabor features, multilevel matching. 


\section{INTRODUCTION}

Automatic Palmprint recognition has extensively been researched in recent years and various techniques have been proposed in the literature. Depending on the information they make use of, palmprint recognition techniques can be broadly classified in to three types [2], namely texture, line and appearance based techniques. Most of these techniques are based on ideas from 2D image analysis [1], [3], [4], [5], [6], [7], [8]. Although these methods have been shown to perform well on relatively large databases, efforts are still required to achieve an automated, robust, and high accuracy palmprint recognition system capable of being deployed for high security applications. Often, two-dimensional image-based approaches suffer from changes in imaging factors such as illumination. These inherent limitations can adversely affect the system performance.

Moreover, 2D image based biometric recognition systems are vulnerable to various kinds of attacks. For instance, if one could draw some lines on his palm and make it look like another person's palm, then a 2D palmprint recognition system could easily be circumvented. It may also be possible for an impostor to present a genuine user's fake biometric image to gain access to restricted services. However, the capability of current palmprint authentication systems against such spoof attacks has not yet been investigated in the palmprint literature.

One of the possible solutions to overcome the limitations associated with 2D palmprint authentication systems is to use 3D imaging devices for capturing surfaces of human palm and use this data in performing user identification. Such observations are relatively invariant to illumination and provide more information on depth and curvature of lines and wrinkles on the palm surface. 
There are a few works in the field of hand geometry recognition that exploits 3D features of the hand/finger. Even though a typical hand geometry recognition system [15], [16] utilizes 3D features such as height of the finger and the palm, these systems rely entirely on intensity images of the hand for feature extraction. Hand geometry recognition system described in [17] uses a simple peg free imaging set up, but does not make use of any 3D features of the hand. The authors in [18] investigated the use of finger back surface as a biometric feature. A high end range scanner is used to capture the range image of the back of the hand. They report a recognition rate of $99.4 \%$ using data obtained on the same day, while it reduced to $74 \%$ when gallery and probe images were obtained on different days. The US patent [29] illustrates the process of acquisition of 3D finger and palmprint information using multi camera and light projection system. However, this patent does not describe any method for verification/ recognition using the acquired biometric information. Malassiotis et al. [19] have developed a biometric authentication system based on measurements of user's 3D hand geometry. A low-cost 3D sensor is used to capture the range image of the user's hand. Experiments on a database of 73 subjects achieved an EER of 3.6\%. Furthermore, in order to improve the performance, biometric researchers have combined 2D and 3D features for face [13], [14] and ear biometrics [14]. However, there has not been any previous study in the palmprint literature to investigate the utility of 3D palmprint features. This has motivated us to explore the utility of $3 \mathrm{D}$ palmprint features [28] and the possibility of combining them with $2 \mathrm{D}$ features for palmprint recognition. Major contributions of this paper can be summarized as follows:

1) This paper, for the first time, investigates the utility of $3 \mathrm{D}$ palmprint features and proposes a new approach for the personal authentication based on 3D 
palmprint images of the user. The surface curvature based features are employed to represent the information in the captured 3D palmprint images, while normalized local correlation is used to match extracted features.

2) We propose a multilevel framework for personal authentication that efficiently combines 2D and 3D palmprint features. At the first level, 3D features are matched. If the resulting match score is above a particular threshold, the query is rejected as a fake palm or an impostor and the matching process is terminated at Level 1. Otherwise matching proceeds to Level 2 where 2D and 3D matching scores are combined to decide if the user is a genuine or impostor. The experimental results presented in this paper demonstrate that the combination of $2 \mathrm{D}$ and $3 \mathrm{D}$ matching scores, using the proposed multilevel matcher, results in significant performance improvement over the case when either 2D or 3D features are employed alone.

3) We investigate the robustness (against spoof attacks) of a 2D palmprint image based system and present experimental results to demonstrate the utility of 3D palmprint features for enhancing the robustness of the proposed system. A robust biometric system has to deal with a number of security threats. Among various kinds of attacks [22], one of the simplest ways to attack a biometric system is to present a fake biometric to the acquisition device. Researches have shown that biometric traits such as fingerprint [24] and gait [25] are vulnerable to spoof attacks. As a result, various approaches have been proposed for fake fingerprint detection [26], [27]. However, no attempt has been made so far to analyze the vulnerability of palmprint systems to such attacks. 
The organization of rest of this paper is as follows; section 2 describes the operation of the proposed system with a block diagram. This section also details the 3D acquisition device that captures both an intensity image of the palmprint and its 3D structure. In section 3, first we briefly describe the $2 \mathrm{D}$ feature extraction using the competitive coding scheme, followed by 3D feature extraction in detail. Section 4 describes the feature matching techniques employed in our system. Experimental results and discussions are presented in section 5. Section 6 concludes this work with comments on future research.

\section{PROPOSED SYSTEM}

We propose a palmprint verification system utilizing both $2 \mathrm{D}$ and $3 \mathrm{D}$ features of human palm. Our goal is to improve the performance of existing $2 \mathrm{D}$ image based system. Block diagram of the proposed biometric system is shown in Figure 2. The image acquisition device, developed at Biometric Research Center at the Hong Kong Polytechnic University, is based on the principle of structured light. Figure 4 shows acquisition of a sample hand image using the developed device. Infrared sensors are employed to detect the presence of the hand on the acquisition device (see Figure 1). When a hand is detected, the device projects multiple light patterns onto the palm surface and acquires depth information using active triangulation. In order to distinguish between stripes, they are coded with different brightness. The system uses a computer controlled Liquid Crystal Display (LCD) projector that can generate arbitrary stripe patterns. A CCD camera is used to capture the images formed on the object. The sequence of images captured by the CCD camera is processed by the algorithm running on the associated device to obtain the 3D palm data. This device 
acquires $3 \mathrm{D}$ data in point cloud form. It also acquires a registered intensity image in the course of its normal operation. The size of these images are set to $768 \times 576$ pixels.

Each of the acquired 2D images are processed to extract the region of interest (ROI) using the method described in reference [8]. This method establishes a coordinate system with the gaps between the fingers as reference points and extracts a subimage of fixed size located at the central part of the palmprint. Since the acquired $2 \mathrm{D}$ and the $3 \mathrm{D}$ images are registered, the pixel coordinates of the $2 \mathrm{D}$ subimage can be used to locate the ROI and extract the $3 \mathrm{D}$ subimage.

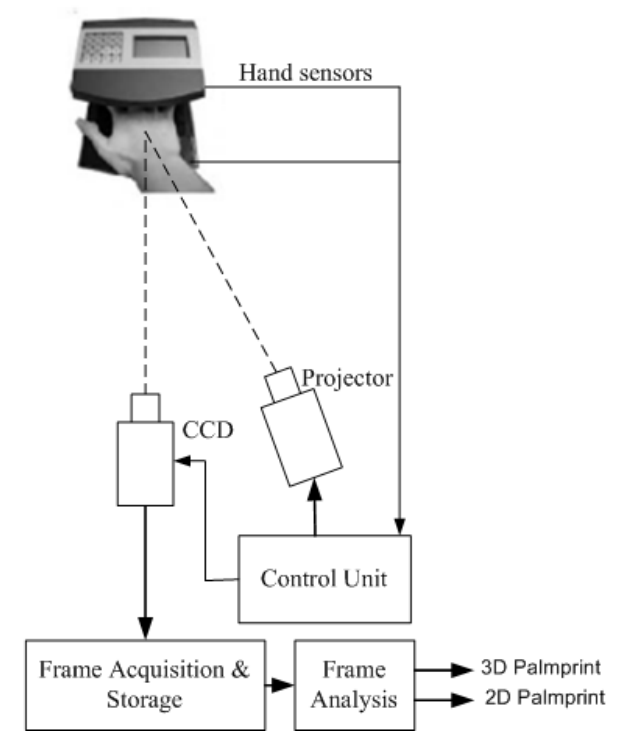

Figure 1. Block diagram of the image acquisition module

Figure 3 shows samples of 3D and corresponding 2D subimages in our database. These $3 \mathrm{D}$ and $2 \mathrm{D}$ subimages are further processsed to extract surface curvature (3D palmprint feature) and competitive code (2D palmprint feature) [9] respectively. Similarity between two curvature maps are then calculated using local correlation method. If the matching score is greater than the threshold of Decision Module I, the query is rejected as a fake palm or an impostor and the process is terminated. This constitutes Level 1 of the proposed multilevel authentication approach. On the other hand, if the matching score computed at Level 1 is below the threshold, matching proceeds to Level 2, where 
the matching scores from 2D palmprint features are combined with the $3 \mathrm{D}$ matching scores from Level 1 to obtain a final matching score. This score is used to make a decision as to whether the claimant is a genuine user or impostor. The entire process of our system is illustrated in Figure 2.

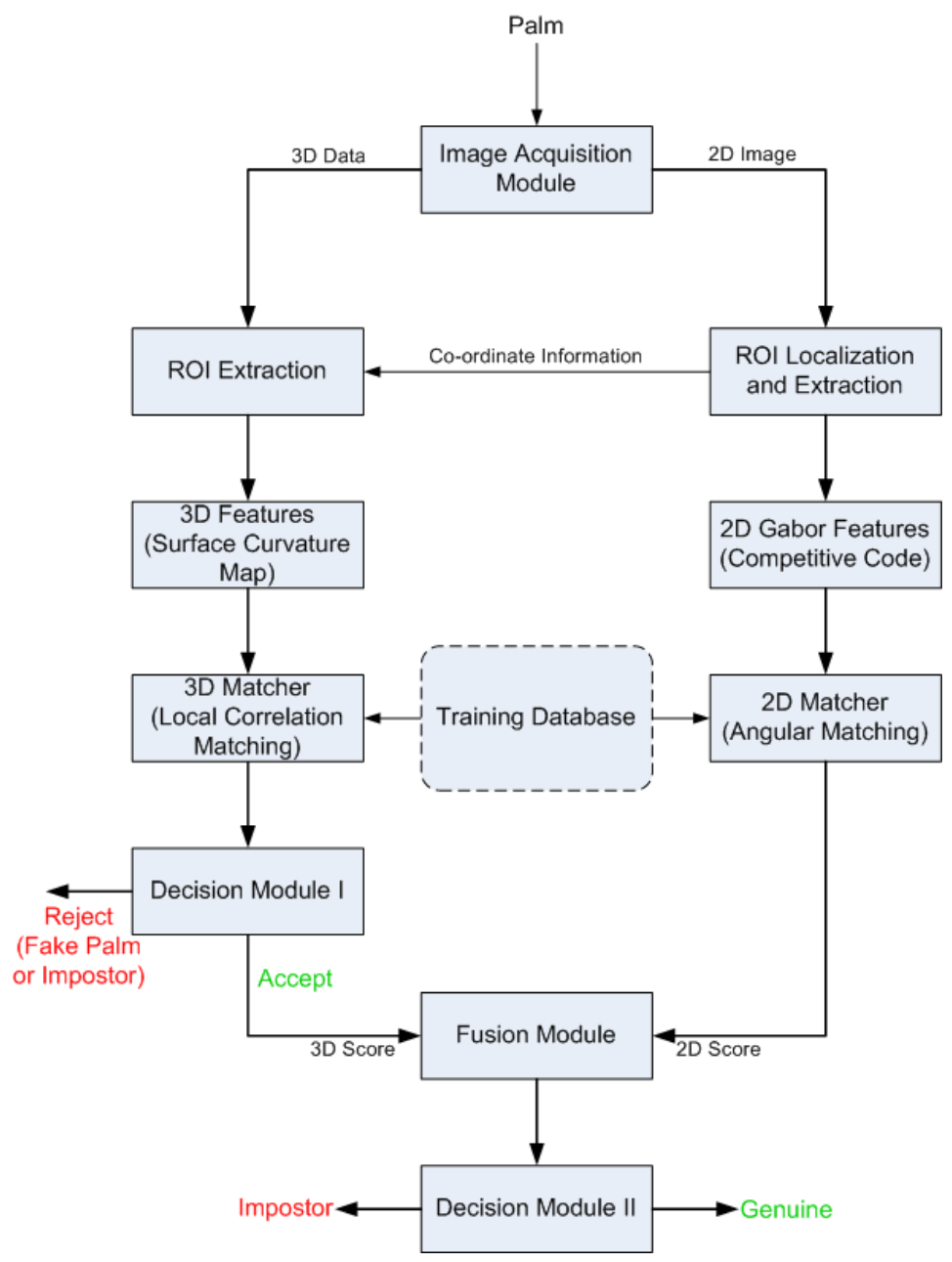

Figure 2. Block diagram of the proposed palmprint authentication system.
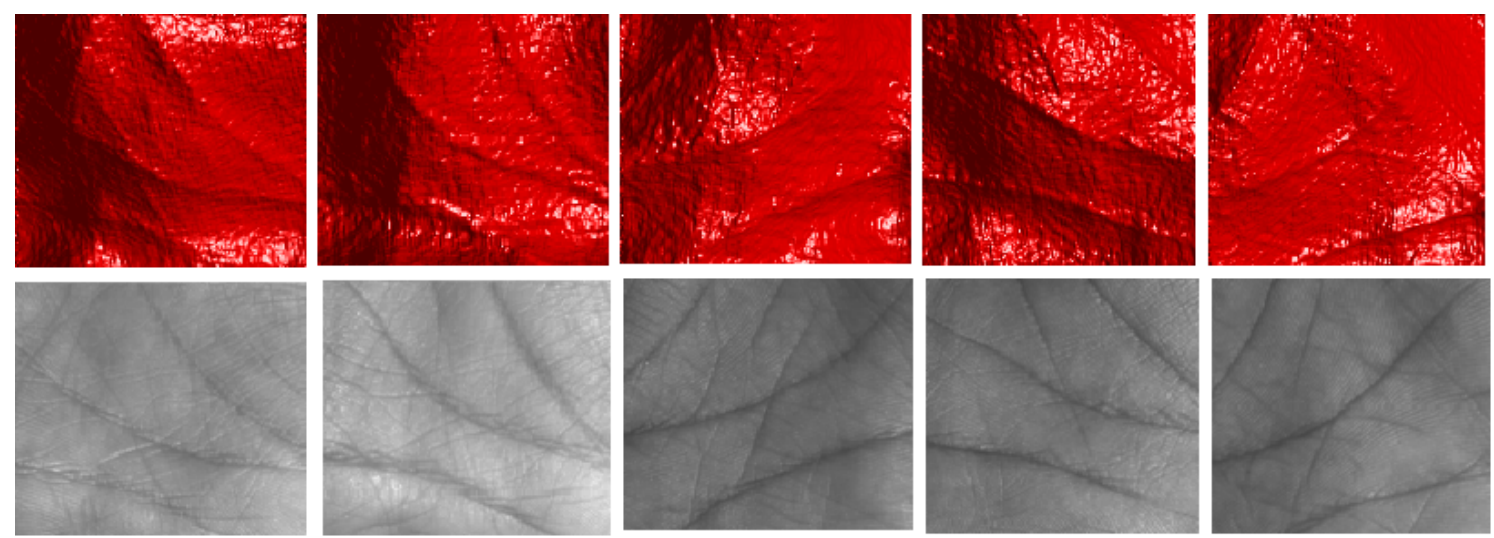

Figure 3. Samples of 3D and 2D palmprints in the database 


\section{FEATURE EXTRACTION}

\subsection{EXRACTION OF 2D PALMPRINT FEATURES}

Competitive coding scheme [9] has been one of the best performing feature extraction method for palmprint verification. This coding scheme uses the neurophysiology based 2D Gabor filters to extract orientation information from the palmlines. A family of 2D Gabor wavelets, satisfying wavelet theory and the neurophysiological constraints can be derived from general complex 2D Gabor function as [23]:

$$
\psi\left(x, y, \omega_{o}, \theta\right)=\frac{\omega_{o}}{\sqrt{2 \pi} \kappa} e^{\frac{-\omega_{o}^{2}}{8 \kappa^{2}}\left(4(x \cos \theta+y \sin \theta)^{2}+(-x \sin \theta+y \cos \theta)^{2}\right)}\left[e^{i\left(\omega_{o} x \cos \theta+\omega_{o} y \sin \theta\right)}-e^{\frac{-\kappa^{2}}{2}}\right]
$$

where $\omega_{o}$ is the radial frequency in radians per unit length and $\theta$ is the wavelet orientation in radians. $\kappa$ is a constant, value of which depends on the frequency bandwidth. The center frequency $\omega_{o}$ of the Gabor filters can be derived to be $\frac{\kappa}{\sigma}[23]$. Each of the extracted subimages is convolved with Gabor filters oriented in six different directions and a competitive rule is formulated to select the prominent orientation, $\theta_{p}$. This orientation is selected to be the one with minimum filter response and is defined as:

$p=\arg \min _{j}\left(I(x, y) * \psi_{R}\left(x, y, \omega, \theta_{j}\right)\right)$

where $I$ is a $2 \mathrm{D}$ subimage, $\psi_{R}$ represents the real part of the Gabor filter, and '*, denotes discrete convolution. Orientations of the filters are chosen to be $\theta_{j}=j \pi / 6, j=\{0,1, \ldots, 5\}$. The winning orientation from Equation 2 is binary coded using 3 bits and stored as a feature. A detailed description of this approach has been detailed in our earlier work [9]. 


\subsection{EXRACTION OF 3D PALMPRINT FEATURES}

The captured 3D palmprint images are rich in local structural features. Therefore, the surface curvature maps, which uniquely characterize the local shape of a surface, are used as 3D representations. The surface curvature properties have been widely used to represent and recognize various surfaces. Properties such as mean and Gaussian curvatures are also used to classify points on a surface to different classes $[21]$

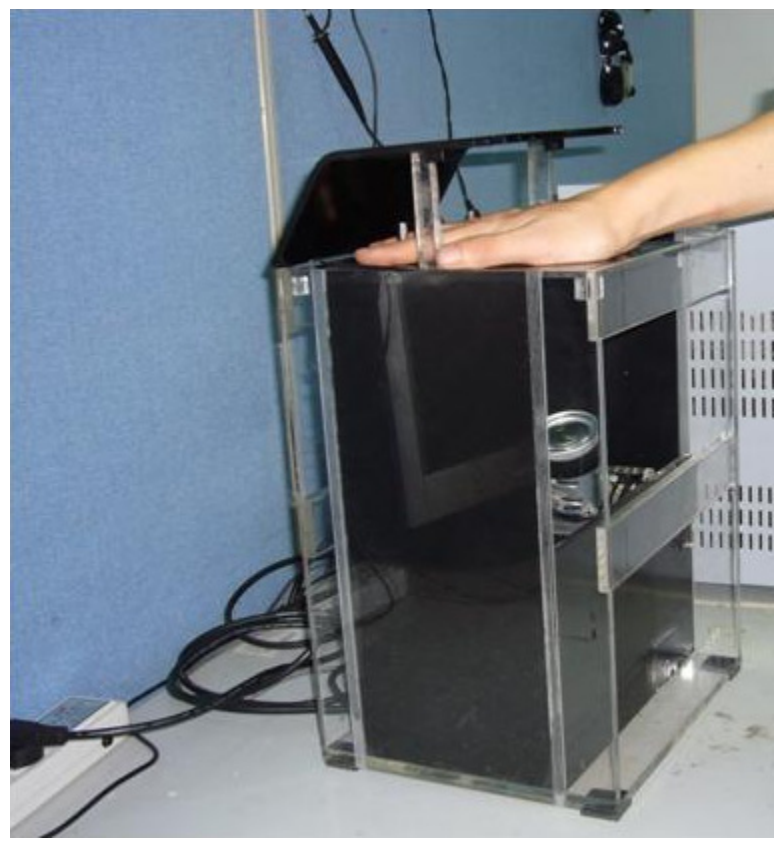

Figure 4. Data acquisition using the developed device.

For a surface patch represented by $\mathrm{X}(u, v)=(u, v, f(u, v))$, Gaussian curvature $(K)$ and mean curvature $(H)$ are calculated as follows [10]:

$K(X)=\frac{f_{u u} f_{v v}-f_{u v}^{2}}{\left(1+f_{u}^{2}+f_{v}^{2}\right)^{2}} \quad$ and $H(X)=\frac{\left(1+f_{u}^{2}\right) f_{v v}+\left(1+f_{v}^{2}\right) f_{u u}-2 f_{u} f_{v} f_{u v}}{\left(1+f_{u}^{2}+f_{v}^{2}\right)^{3 / 2}}$

where, $f_{u}, f_{v}$ and $f_{u u}, f_{v v}, f_{u v}$ are first and second order partial derivatives of $f(u, v)$. Once the values of $K$ and $H$ are found at every point on the 3D surface, the principal curvatures $k_{1}$ and $k_{2}$ can be determined as: 


$$
k_{1}, k_{2}=H \pm \sqrt{H^{2}-K}
$$

In practice, these principal curvature values at every pixel are computed by fitting a quadratic surface over a local neighborhood and then estimating first and second derivatives of the surface. In our experiments, the partial derivatives are estimated using derivative operators [20]. This approach employs a local least squares surface fit using orthogonal polynomial basis functions. Since this process involves the estimation of second derivates, the estimated curvature values are sensitive to noise in the input $3 \mathrm{D}$ data. This problem can be overcome by increasing the size of the local neighborhood used for fitting the surface. However, a large window size can smooth the image, resulting in the loss of minute details in the $3 \mathrm{D}$ palmprint image. In our experiments, we empirically fixed the size of window as $13 \times 13$.

To represent the curvature of every point on the 3D palmprint image by a scalar value, we utilized the curvedness $(C)$ introduced in [11]. The positive value $\mathrm{C}$ is a measure of how sharply or gently curved a point is [21]. It is defined in terms of principal curvatures $k_{1}$ and $k_{2}$, as:

$$
C=\sqrt{\left(k_{1}^{2}+k_{2}^{2}\right) / 2}
$$

Thus a scalar value of curvature is obtained for every point on the $3 \mathrm{D}$ palmprint image and this can be stored in a 2D matrix or an image. In this paper, set of such scalar values is referred to as surface curvature map. Figure 5 shows curvature maps for some of the 3D palmprint images in our database. It can easily be observed that the surface curvature maps obtained by the proposed feature extraction algorithm closely resembles the palmlines, especially the strong principal lines. 

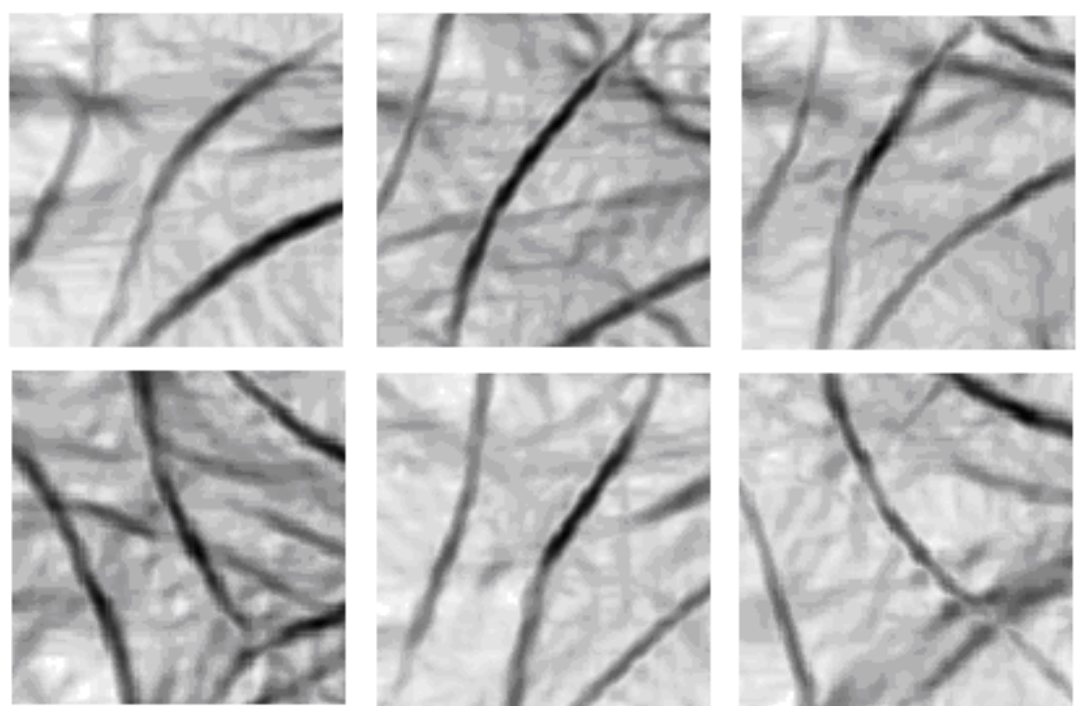

Figure 5. Surface curvature maps for six different subjects

\section{FEATURE MATCHING}

\subsection{MATCHING 2D FEATURES}

Angular distance described in [9] is employed for comparing features extracted from 2D palmprint images. Let $P$ and $Q$ be the two feature matrices (competitive codes) and $P_{M}$ and $Q_{M}$ be the corresponding masks used for indicating the non palmprint pixels. Angular distance $D(P, Q)$ is defined by the following equation:

$$
D(P, Q)=\frac{\sum_{y=0}^{N} \sum_{x=0}^{N} \sum_{i=0}^{3}\left(P_{M}(x, y) \cap Q_{M}(x, y)\right) \cap\left(P_{i}^{b}(x, y) \otimes Q_{i}^{b}(x, y)\right)}{3 \sum_{y=0}^{N} \sum_{x=0}^{N} P_{M}(x, y) \cap Q_{M}(x, y)}
$$

where $\cap$ and $\otimes$ denote the bitwise AND and XOR operations respectively. $P_{i}^{b}\left(Q_{i}^{b}\right)$ is the $i^{\text {th }}$ bit plane of $P(Q)$. Taking into account the possible translations in the extracted subimage (with respect to the one extracted during the enrolment), multiple matchings are performed with one of the features translated in horizontal and vertical directions. Minimum of the resulting matching scores is considered to be the final score. 


\subsection{MATCHING CURVATURE MAPS}

The inputs for the matching algorithm are two sets of curvature maps of size 128 $\times 128$. The feature matching process establishes the similarity between the gallery and the test templates. In our work, we employ a image matching technique, normalized local correlation for comparing two curvature maps. Result of this matching is a correlation value for every point in the input curvature maps. Average of these correlation values is considered to be the matching score. The expression for normalized local correlation is given by:

$$
C=\frac{\sum_{i=-N}^{N} \sum_{j=-N}^{N}\left(P_{i j}-\bar{P}\right)\left(Q_{i j}-\bar{Q}\right)}{\sqrt{\left[\sum_{i=-N}^{N} \sum_{j=-N}^{N}\left(P_{i j}-\bar{P}\right)^{2}\right]\left[\sum_{i=-N}^{N} \sum_{j=-N}^{N}\left(Q_{i j}-\bar{Q}\right)^{2}\right]}}
$$

where $P_{i j}$ and $Q_{i j}$ are curvature values in the neighborhood of the points being matched in gallery and test curvature maps respectively, and $\bar{P}$ and $\bar{Q}$ are the mean curvature values in those neighborhoods. $(2 N+1) \times(2 N+1)$ is the size of the neighborhood in pixels. Clearly, the value of $C$ lies in the range of $[-1,1]$ with values 1 and -1 indicating a perfect match and mismatch respectively. Figure 6 and 7 illustrate the process of matching of two curvature maps of the same and different user respectively. Red (dark) colored pixels in the correlation map represent high values of correlation while blue (light) represents low correlation. Final matching score is the average of pixel values in the correlation map. It can be observed from Figure 6 that genuine matching results in a correlation map with large regions of red colored pixels, indicating high correlation between the two curvature maps being matched. 


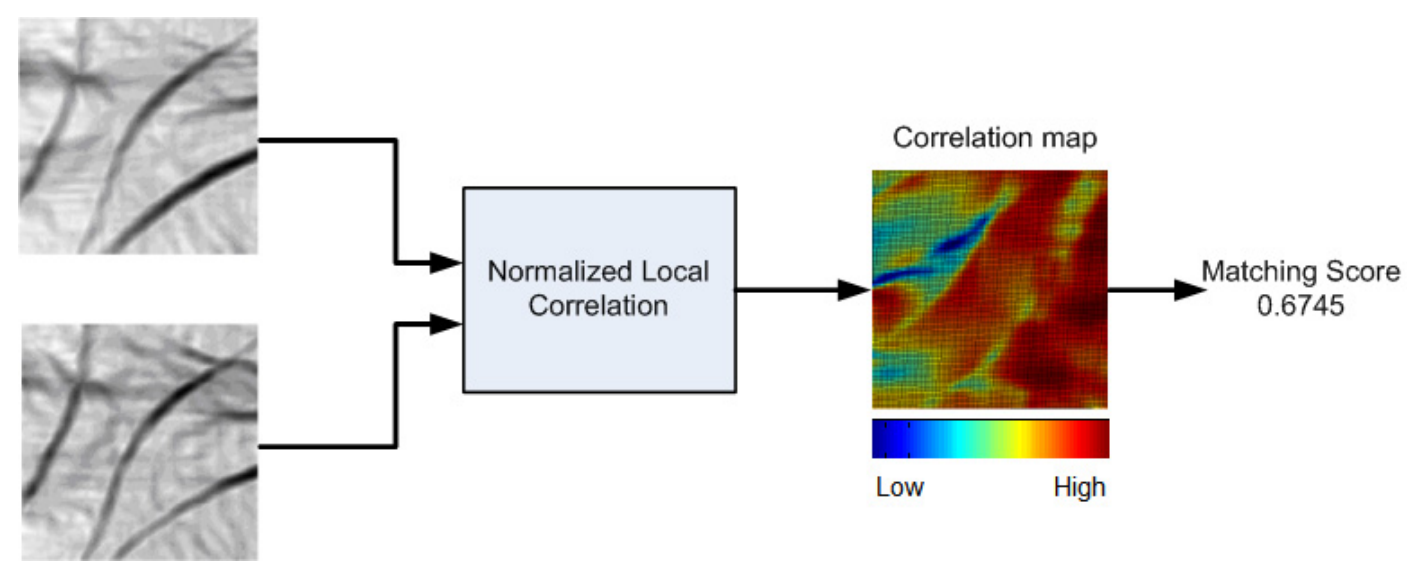

Figure 6. Matching of two curvature maps from the same subject

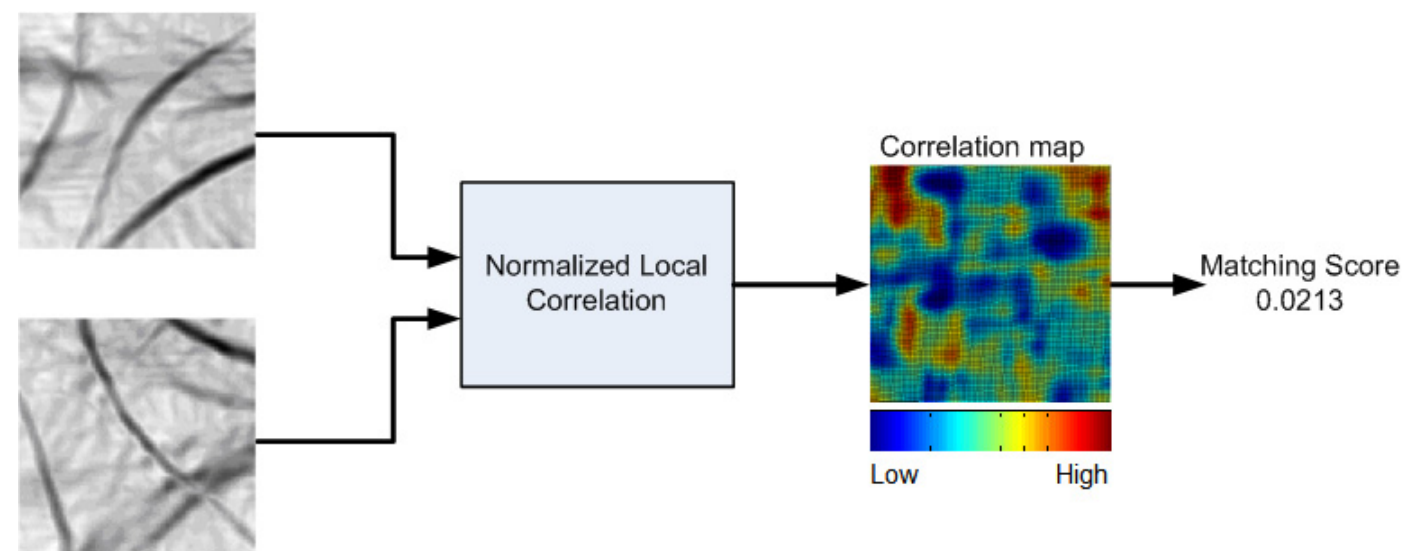

Figure 7. Matching of two curvature maps from different subjects

\section{EXPERIMENTAL RESULTS AND DISCUSSION}

\subsection{VERIFICATION EXPERIMENTS}

In order to evaluate the performance of the proposed system, we performed verification experiments on a database of 108 subjects. This database was collected at the Biometric Research Center, the Hong Kong Polytechnic University over a period of two months. The database was collected in two sessions, with an interval of 2 weeks and mainly consisted of volunteers and students from our university. Ideally, the database should include some fake palmprints to test the robustness of the proposed system. However, in this set of experiments, we focus on analyzing 2D and 3D palmprint features. The main objective was to achieve performance improvement 
by combining $2 \mathrm{D}$ and $3 \mathrm{D}$ palmprint features that are simultaneously acquired from our imaging setup. However, the utility of our approach is not only limited to the performance improvement. In the next section, we illustrate the experiments performed to analyze the robustness of the 3D palmprint features against spoof attacks. The database for the experimental results reported in this section includes only real palmprint images. All images were acquired using the capture device shown in Figure 4. For each subject, six samples of 2D and 3D palmprint images were captured and stored in the database. Thus, there are a total of 648 palmprint images in our database. To obtain the verification accuracy of the proposed method, each palmprint image is matched with all other palmprint images in the database, resulting in 1,620 genuine and 208,008 impostors matching scores for each of the two modalities. Figure 8 shows a scatter plot of the genuine and impostor score distributions obtained from 2D and 3D palmprint features. It can be observed that the two distributions are well separated and a linear classifier would be able to discriminate the genuine and impostor classes.

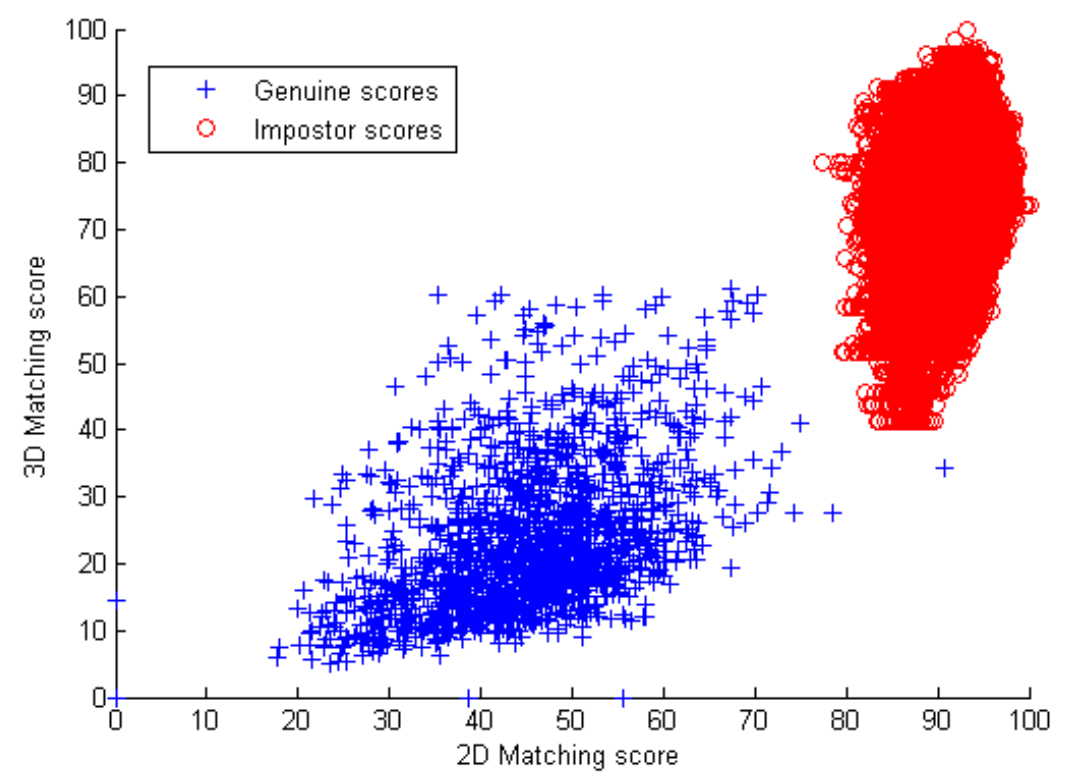

Figure 8. Genuine and impostor scores in 2 dimensional space 
We first analyzed the individual achieved performance from 2D and 3D palmprint representation. Figure $9(a)$ and $9(b)$ show the performance, in terms of False Acceptance Rate (FAR) and False Rejection Rate (FRR) characteristics obtained from 2D and 3D features respectively. The Equal Error Rate (EER) achieved from the two

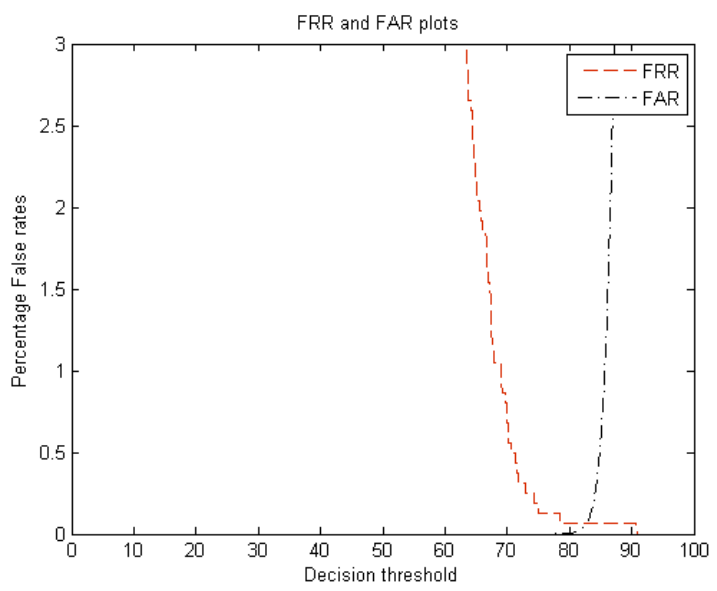

Figure 9(a). FAR and FRR plots for 2D features

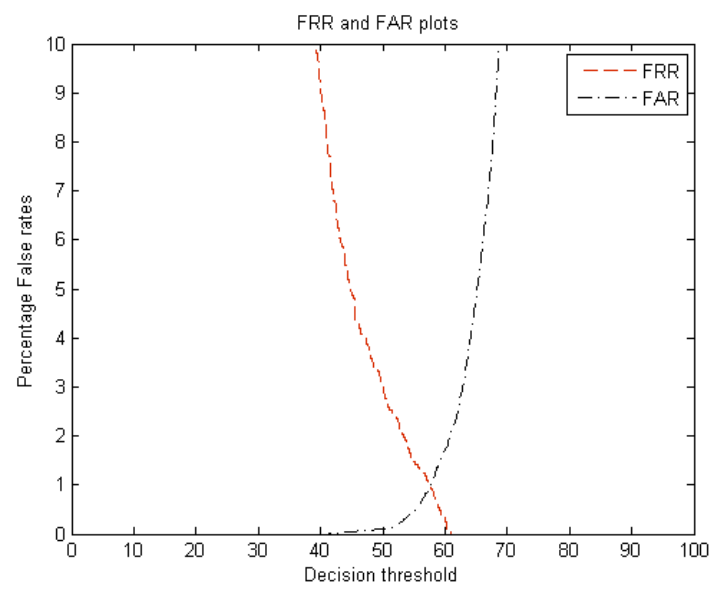

Figure 9(b). FAR and FRR plots for 3D features separate experiments using 2D and 3D features is illustrated in Table 1. In Figures 9a and $9 \mathrm{~b}$, it is important to note that the $2 \mathrm{D}$ palmprint representation clearly outperforms 3D in terms accuracy.

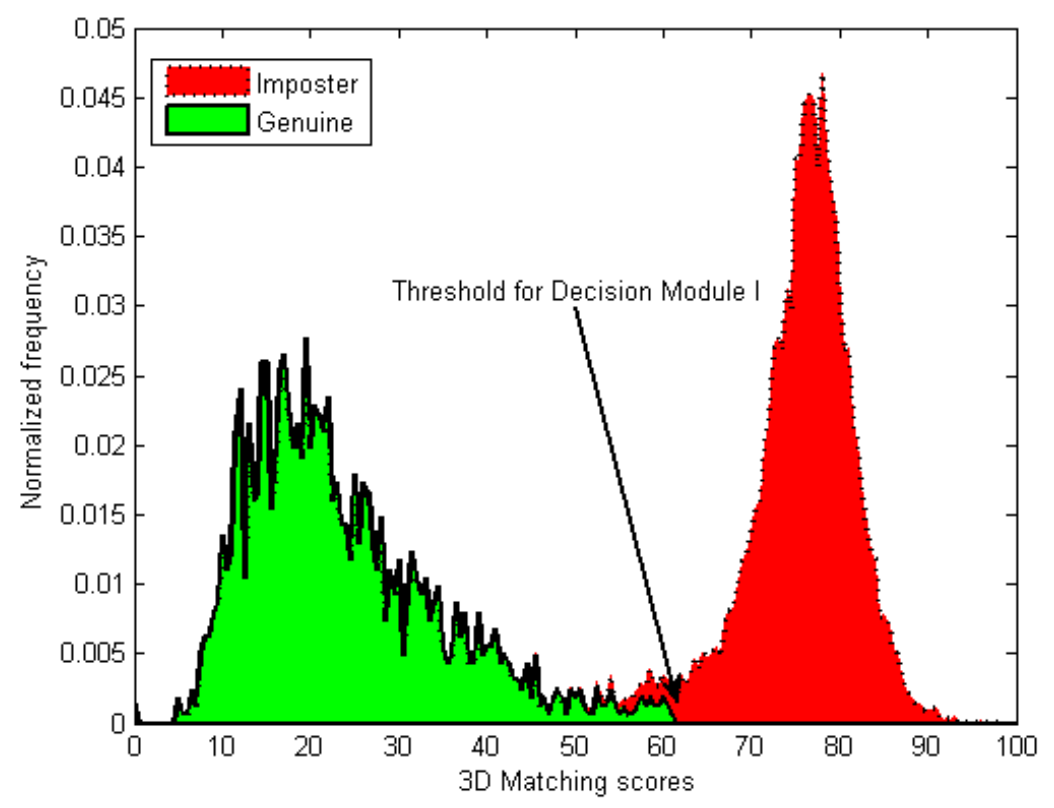

Figure 10. Illustrates the selection of threshold for Decision Module I 
Further experiments were carried out to ascertain the possible performance improvement by combining two $(2 \mathrm{D}+3 \mathrm{D})$ palmprint representations using the proposed multilevel matching framework. The threshold of the Decision Module I was selected such that no genuine users are rejected at Level 1. This can be achieved by setting the threshold to the operating point at which FRR for 3D palmprint features becomes zero. The value of this threshold was found to be 61.03 (see Figure 10). All 3D matching scores (genuine as well as impostor) above this threshold are rejected at Level 1. After rejection at Level 1, there were 1620 genuine and 203,667 impostor scores for each of the two modalities. These scores were carried over to the next level. At this level (Level 2), since the genuine and impostor distributions are originally well separated (see Figure 8), we employ a simple weighted sum rule to combine the 2D and 3D matching scores. The combined score (Level 2 match score) can be expressed as:

$$
S_{2 D+3 D}=w_{1} S_{2 D}+w_{2} S_{3 D}
$$

where $S_{2 D}$ is the $2 \mathrm{D}$ matching score normalized to $(0,100)$ range. The $3 \mathrm{D}$ matching score, which in fact is a dissimilarity score, is firstly normalized to $(0,100)$ range and then converted to a similarity score to obtain $S_{3 D}$. The weights $w_{1}$ and $w_{2}$ are tuned to provide the best verification results. The optimal values of $w_{1}$ and $w_{2}$ were empirically calculated and found to be 0.56 and 0.44 respectively.

Results of our experiments are summarized in Table 1 . Decidability index $\left(d^{\prime}\right)$ is used as a measure to quantify the improvement in the separability of impostor and genuine matching score distributions. It is computed as:

$$
d^{\prime}=\frac{\left|\mu_{1}-\mu_{2}\right|}{\sqrt{\frac{\sigma_{1}^{2}+\sigma_{2}^{2}}{2}}}
$$


where $\mu_{1}$ and $\mu_{2}$ are the mean values and $\sigma_{1}^{2}$ and $\sigma_{2}^{2}$ are the variances of the genuine and impostor score distributions respectively.

It can be observed from this Table 1 that the proposed multilevel approach for combination of $2 \mathrm{D}$ and $3 \mathrm{D}$ features achieves the best performance, i.e., Equal Error Rate (EER) of $0.0022 \%$ and decidability index of 7.45 . This performance is significantly higher as compared to the case when either 2D or 3D palmprint features alone are used. Figure 11 shows the FAR and FRR plots for the case when both 2D and 2D features are employed.

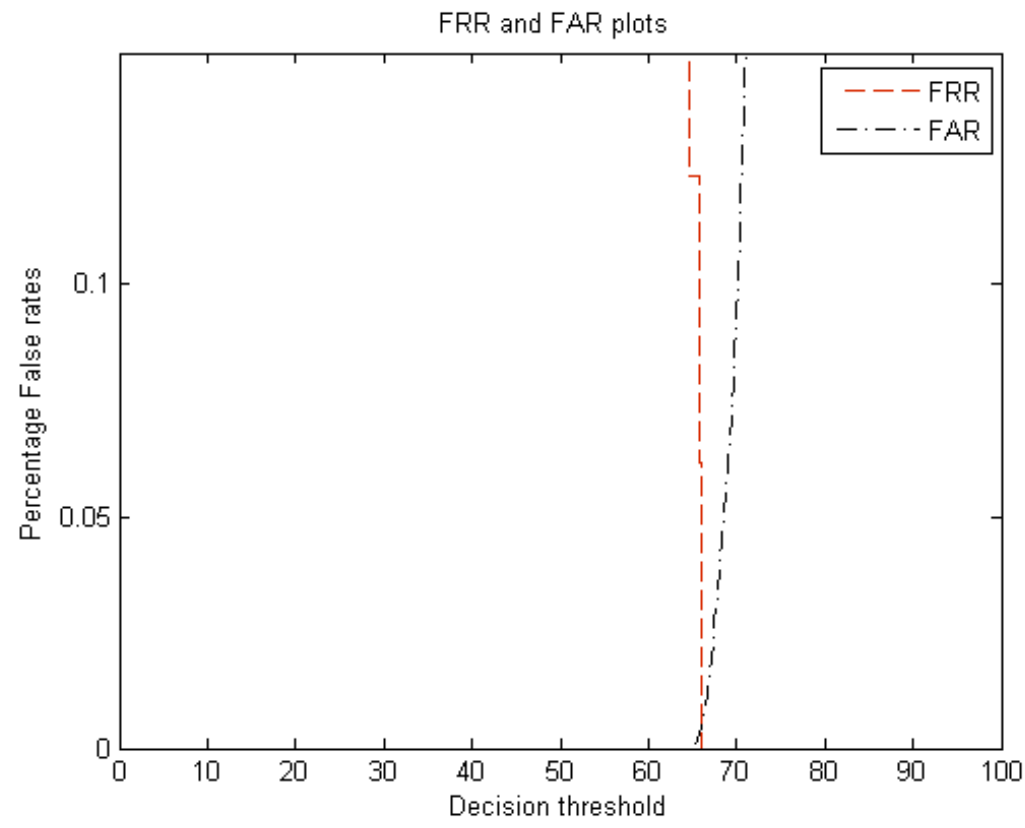

Figure 11. FAR, FRR plots for $(2 \mathrm{D}+3 \mathrm{D})$ features

A comparative Receiver Operating Characteristics (ROC) obtained from the three different set of experiments is shown in Figure 12.

Table 1: Performance indices from 2D, 3D and the (2D+3D) palmprint representations

\begin{tabular}{|c|c|c|}
\hline Palmprint Matcher & EER (\%) & Decidability Index $\left(d^{\prime}\right)$ \\
\hline 2D & 0.0621 & 6.50 \\
\hline 3D & 0.9914 & 5.97 \\
\hline Multi level (2D+3D) & 0.0022 & 7.45 \\
\hline
\end{tabular}




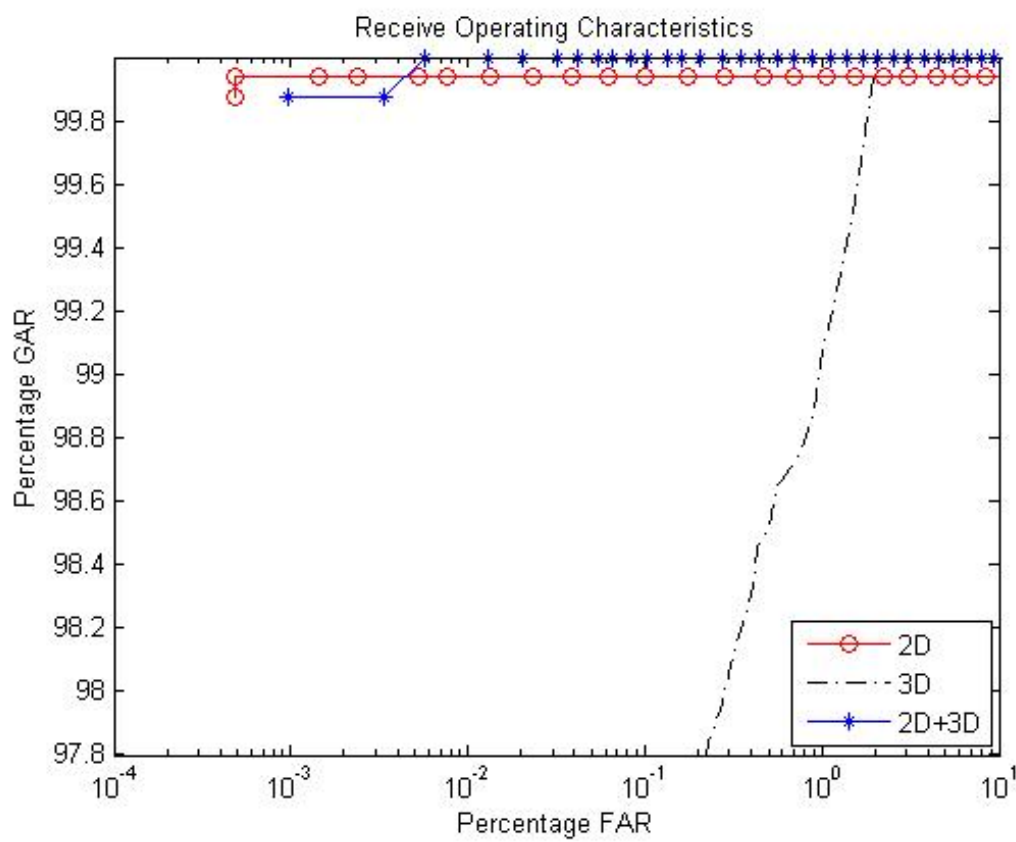

Figure 12. The ROC curves for $2 \mathrm{D}, 3 \mathrm{D}$ and the proposed multilevel $(2 \mathrm{D}+3 \mathrm{D})$ features

\subsection{SPOOF EXPERIMENTS}

In this set of experiments, we investigate the robustness of the $3 \mathrm{D}$ palmprint features against the sensor level spoof attacks. We also analyze the vulnerability of 2D image based authentication system against such attacks. Our experiments involved collecting data from five subjects, in two stages. In the first stage, subjects were asked to present their "real" hand, while in the second, images of the same users' hand (palm side) printed on a paper were presented to the capture device. Figure 13 shows fake palmprints used for our experiments. The fake palmprints generated from genuine users' palmprint images (Figure 13(a)-(d)) were simply pasted on one real palm, as shown in Figure 13(e). This real hand, with fake palmprint, was presented to the developed system that can acquire both 2D and 3D palmprint images. Again, experiments were performed in a verification scenario, i.e., a user's palmprint image captured in the first stage is matched to the one captured in the second stage. A match is counted as correct if the resulting matching score is less than the system threshold. 
For the purpose of analysis we consider this threshold to be the operating point of EER as it represents commonly preferred operating point for most of the practical deployments. The results from this set of experiments are summarized in Table 2 .

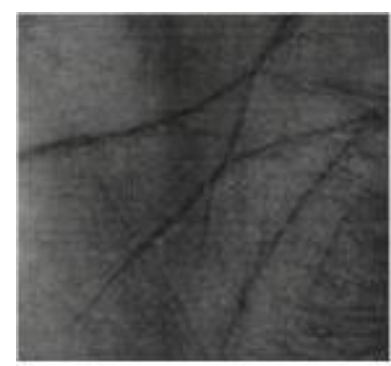

(a)

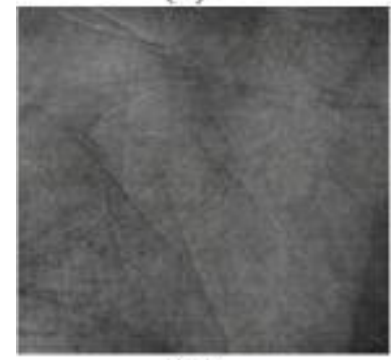

(c)

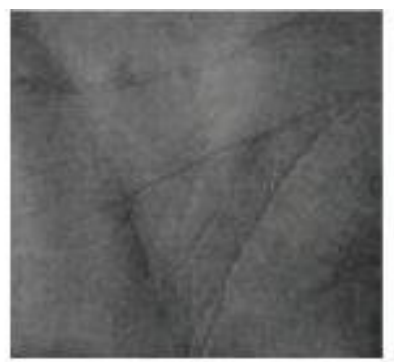

(b)

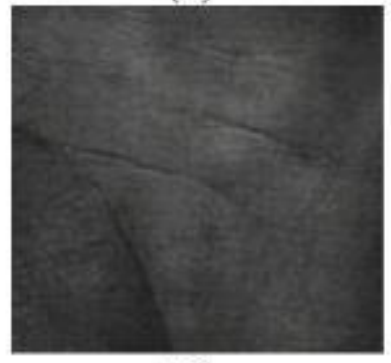

(d)

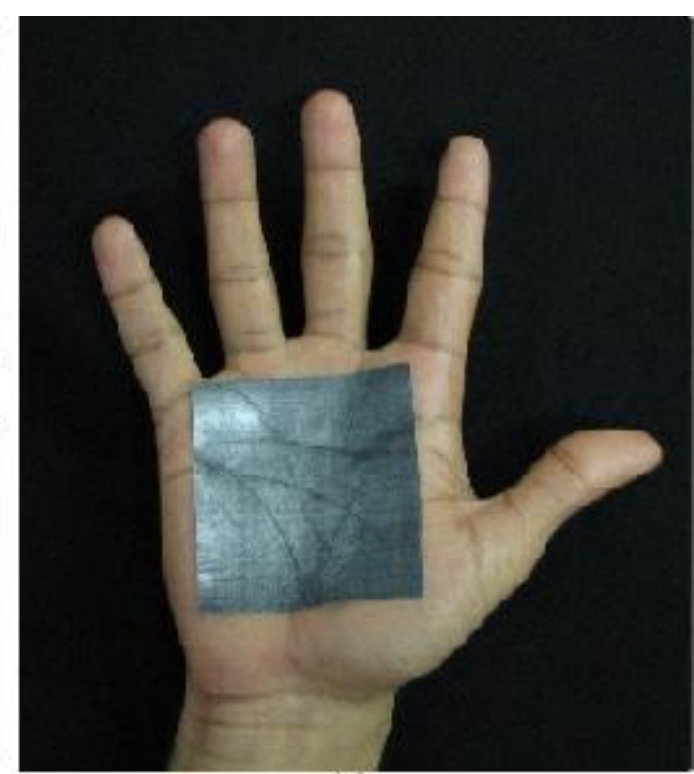

(e)

Figure 13. Fake palmprints. (a) - (d) Show the palmprint images printed on the paper to use as spoofs. (e) Shows a fake palmprint pasted on the hand to spoof the system.

Table 2: Matching scores from fake palmprints (spoof attack analysis)

\begin{tabular}{|c|c|c|c|}
\hline User ID & $\begin{array}{c}\text { 2D Matching Score } \\
\text { (Threshold = 82.69) }\end{array}$ & $\begin{array}{c}\text { 3D Matching Score } \\
\text { (Threshold = 58.19) }\end{array}$ & $\begin{array}{c}\text { 2D+3D Matching Score } \\
\text { (Threshold = 66.01) }\end{array}$ \\
\hline 1 & 63.75 & 72.42 & 67.56 \\
\hline 2 & 66.69 & 75.68 & 70.65 \\
\hline 3 & 67.09 & 81.89 & 73.60 \\
\hline 4 & 59.46 & 74.59 & 66.10 \\
\hline 5 & 60.26 & 76.79 & 67.5 \\
\hline
\end{tabular}

The experimental results in Table 2 show that the user verification using 2D

palmprint features fails to discriminate between real and fake palmprint samples for all users. As seen from the Table 2, matching scores for 2D palmprint features are well below the threshold at EER, which suggest that the system considers all matches as correct. On the other hand, Table 2 indicate that for the 3D and $(2 \mathrm{D}+3 \mathrm{D})$ features, the system would count all matches as incorrect or negative as the matching scores for $3 \mathrm{D}$ and $2 \mathrm{D}+3 \mathrm{D}$ are above their corresponding thresholds. In fact, 3D performs better 
than $2 \mathrm{D}+3 \mathrm{D}$ (matching scores for $3 \mathrm{D}$ are well above the threshold, while $2 \mathrm{D}+3 \mathrm{D}$ scores are closer to the threshold). This is why we rely entirely on $3 \mathrm{D}$ features to reject fake palmprints at Level 1 of the proposed multilevel matching algorithm. It may be noted that the threshold for Decision Module I was set to 61.03, and all the 3D match scores in Table 2 are above this threshold. This suggests that the proposed system will be successful in rejecting all fake palms at Level 1. The experimental results presented in Table 2 demonstrate the utility of 3D palmprint features for enhancing the robustness (against spoof attacks) of the proposed system.

\section{CONCLUSIONS}

In this paper, we present experimental results from a newly developed palmprint authentication system that can simultaneously acquire and combine $2 \mathrm{D}$ and $3 \mathrm{D}$ palmprint features. This paper also presented the comparative performance evaluation of $2 \mathrm{D}$ and $3 \mathrm{D}$ palmprint representations. Experimental results on a real palmprint database from 108 subjects demonstrate that with proper selection of weight parameters of fusion scheme, significant performance improvement (over 2D and 3D alone) can be achieved. The experimental results presented in section 5 illustrate a performance gain of $96 \%$ in EER and $14.6 \%$ in Decidability Index when the simultaneously acquired 3D features are combined with traditional 2D palmprint features. Experimental results presented in section 5.1 suggest that the performance of palmprint authentication using 3D features alone is promising. However, our comparative analysis, where 2D features achieve lower error rates compared to the $3 \mathrm{D}$ features, suggests that the $2 \mathrm{D}$ features are more discriminative than $3 \mathrm{D}$ features for palmprint representation. 
The experiments performed in section 5.2, to investigate the robustness of $2 \mathrm{D}$ image based approaches, expose the vulnerability of such systems to spoof attacks. On the other hand, palmprint authentication systems based on 3D features, as well as combination of $(2 \mathrm{D}+3 \mathrm{D})$ features, utilize depth information from the palm surface, therefore making them extremely difficult to circumvent. It may also be noted that the proposed system is more robust to noise such as some text or lines drawn on the palmprint. This is because the $3 \mathrm{D}$ depth features extracted from such palms are unaffected by noise and therefore results in a more robust system compared to the $2 \mathrm{D}$ image based palmprint authentication systems. Our experiments also show that $2 \mathrm{D}$ palmprint features can be more prone to spoof attacks, compared to fingerprint systems that require special materials and sometimes special skills. Therefore there is an urgent need to develop robust palmprint authentication techniques capable of withstanding such attacks.

The experimental results reported in this paper led us to believe that the proposed system effectively combines the high discriminatory power of $2 \mathrm{D}$ and robustness of 3D palmprint representations, resulting in a biometric system that has the potential to be deployed for security critical applications. Future research should be directed towards developing better 3D palmprint representations and exploring different fusion strategies. Though the developed system has been shown to be robust against spoof attacks, it is still possible (though extremely difficult) to fabricate fake 3D palm models to circumvent the system. Therefore, another area of future research is to develop techniques for fake palmprint detection. Now that the performance (accuracy as well as robustness) of the proposed system has been investigated on a moderate sized database, it would be interesting to test its performance on a very large user population, i.e. scalability of the system. 


\section{REFERENCES}

[1] P. H. Hennings-Yeomans, B. V. K. Vijaya Kumar, and M. Savvides, "Palmprint classification using multiple advanced correlation filters and palm-specific segmentation," IEEE Trans. Inf. Forensics Security, vol. 2, no. 3, pp. 613-622, Sep. 2007.

[2] A. Kumar and D. Zhang, "Personal authentication using multiple palmprint representation", Pattern Recognition, vol. 38, no. 10, pp. 1695-1704, Oct. 2005.

[3] T. Connie, A. T. B. Jin, M. G. K. Ong, and D. N.C Ling, "An automatic palmprint recognition system, "Image and Vision Computing, vol. 23, no. 5, pp. 501-515, May 2005.

[4] A. Kumar, D. C. M. Wong, H. C. Shen, and A. K. Jain, "Personal verification using palmprint and hand geometry biometric," Proc. 4 th AVBPA, Guildford, U.K., pp. 668-675, 2003.

[5] W. Li, D. Zhang, and Z. Xu, "Palmprint identification by fourier transform", Int. J. Patt. Recog. \& Art. Intell. , vol. 16, no. 4, pp. 417-432, 2002.

[6] X. Lu, D. Zhang, and K. Wang, "Fisherpalms based palmprint recognition", Pattern Recognit. Lett., vol. 24, pp. 2829-2838, Nov. 2003.

[7] Z. Sun, T. Tan, Y. Wang, and S. Z. Li, "Ordinal palmprint represention for personal identification," in Proc. CVPR 2005, vol. 1, pp. 279- 284, 2005.

[8] D. Zhang, W. K. Kong, J. You, and M. Wong, "Online palmprint identification”, IEEE Trans. Pattern Anal. Mach. Intell., vol. 25, no. 9, pp. 1041-1050, Sep. 2003.

[9] A. W. K. Kong and D. Zhang, "Competitive coding scheme for palmprint verification", in Proc. 17th ICPR, Washington, DC, vol. 1, pp. 1051-4651, 2004.

[10] B. O’Neill, Elementary Differential Geometry. New York: Academic, 1966. 
[11] J. J. Koenderink and A. J. van Doorn, "Surface shape and curvature scales", Image Vision Computing, vol. 10, no. 8, pp. 557-564, Oct. 1992.

[12] K. I. Chang, K. W. Bowyer, and P. J. Flynn, "An evaluation of multimodal 2D+3D face biometrics”, IEEE Trans. Pattern Anal. Mach. Intell., vol. 27, no. 4, pp. 619-624, Apr. 2005.

[13] X. Lu, A. K. Jain, and D. Colbry, "Matching 2.5D face scans to 3D models", IEEE Trans. Pattern Anal. Mach. Intell., vol. 28, no. 1, pp. 31-43, Jan. 2006.

[14] P. Yan and K.W. Bowyer, "Multi-biometrics 2D and 3D ear recognition," in Proc. AVBPA 2005, pp. 503-512, 2005.

[15] A. K. Jain, A. Ross, and S. Pankanti, "A prototype hand geometry-based verification system," in Proc. 2nd AVBPA, Washington, DC, pp. 166-171, 1999.

[16] R. Sanchez-Reillo, C. Sanchez-Avila, and A. Gonzales-Marcos, "Biometric identification through hand geometry measurements," IEEE Trans. Pattern Anal. Mach. Intell., vol. 22, no. 10, pp. 1168-1171, Oct. 2000.

[17] A. Kumar and D. Zhang, "Hand geometry recognition using entropy-based discretization," IEEE Trans. Inf. Forensics Security, vol. 2, no. 2, pp. 181-187, Jun. 2007.

[18] D. L. Woodard and P. J. Flynn, "Finger surface as a biometric identifier," Computer Vision and Image Understanding, vol. 100, no. 3, pp. 357-384, Dec. 2005.

[19] S. Malassiotis, N. Aifanti, and M. G. Strintzis, "Personal authentication using 3D finger geometry," IEEE Trans. Inf. Forensics Security, vol. 1, no. 1, pp. 12 21, Mar. 2006.

[20] P. J. Flynn and A. K. Jain, "On reliable curvature estimation," in Proc. CVPR, pp. 110-116, 1989. 
[21] H. Cantzler and R. Fisher, "Comparison of HK and SC curvature description methods," in Proc. 3 rd Int. Conf. on 3-D Digital Imaging and Modeling, pp. $285-291,2001$.

[22] N. K. Ratha, J. H. Connell, and R. M. Bolle, “An analysis of minutiae matching strength," in Proc. 3 rd AVBPA, pp. 223 - 228, 2001.

[23] T.S. Lee, "Image representation using 2D Gabor wavelet," IEEE Trans. Pattern Anal. Mach. Intell., vol. 18, no. 10, pp. 959 - 971, Oct. 1996.

[24] T. Matsumoto, H. Matsumoto, K. Yamada, and S. Hoshino, "Impact of artificial "Gummy" fingers on fingerprint systems," in Proc. of SPIE, vol. 4677, pp. $275-289,2002$.

[25] D. Gafurov, E. Snekkenes, and P. Bours, "Spoof attacks on gait authentication system," IEEE Trans. Inf. Forensics Security, vol. 2, no. 3, pp. 491-502, Sep. 2007.

[26] A. Antonelli, R. Cappelli, D. Maio, and D. Maltoni, "Fake finger detection by skin distortion analysis," IEEE Trans. Inf. Forensics Security, vol. 1, no. 3, pp. 360-373, Sep. 2006.

[27] S. T. V. Parthasaradhi, R. Derakhshani, L. A. Hornak, and S. A. C. Schuckers, "Time-series detection of perspiration as a liveness test in fingerprint devices," IEEE Trans. Syst., Man, Cybern. C, vol. 35, no. 3, pp. 335-343, Aug. 2005.

[28] V. Kanhangad, D. Zhang, and L. Nan, “A multimodal biometric authentication system based on 2D and 3D palmprint features," in Proc. of SPIE Biometric Technology for Human Identification V, Orlando, vol. 6944, pp. 69440C69440C, Mar. 2008.

[29] F. Chen, "3D Fingerprint and palm print data model and capture devices using multi structured lights and cameras,” U. S. Patent No. 20060120576, 2006. 